\title{
Progress in protein structure and function studies in China during 2010-2011
}

\author{
HU YongLin \\ National Laboratory of Macromolecules, Institute of Biophysics, Chinese Academy of Sciences, Beijing 100101, China
}

Received August 12, 2012; accepted September 16, 2012

Citation: Hu Y L. Progress in protein structure and function studies in China during 2010-2011. Sci China Life Sci, 2012, 55: 927-930, doi: 10.1007/s11427-012-4388-6

The last few years witnessed remarkable progress in the field of protein science research in China, as best illustrated by the increase in both the quality and quantity of publications by Chinese scientists in national and international journals. Here I would like to highlight some of the research and review papers published in China during the 2010-2011 period.

\section{Computational and bioinformatics studies}

Yan et al. [1] studied the impact of different amino-acid networks (such as hydrophobic and hydrophilic networks) on the folding rate of proteins. The paper by Guo et al. [2] proposed an algorithm to predict protein folding rates based on primary amino acid sequences. The results showed that the folding rates could be predicted with relatively high accuracies. Zhang and Luo [3] revealed that protein folding is a quantum conformational transition and that the folding rate parameters can be quantitatively studied based on this observation.

Chen et al. [4] demonstrated that with domain-domain interactions taken into consideration in studies examining protein-protein interactions, human protein interaction networks could provide more detailed information that was distinct from the networks based solely on protein-protein interaction datasets. Such results indicate that domain information should be used to provide additional information

email: yonglin@ibp.ac.cn for understanding human protein interaction networks. Wei [5] constructed an interaction network for integrin-mediated cell adhesion proteins by data-mining. This study revealed a relatively small number of key motifs dominated by three-component complexes.

The paper by Yan et al. [6] indicated that the composition of functional domains can be used to represent proteins in protein fold recognition.

Cong et al. [7] performed extensive multi-conformational docking and molecular dynamics simulations on the possible binding mode of two inhibitors of N-substituted derivatives to the hydrophobic pocket of the HIV-1 envelope protein gp41. The results showed that binding is primarily driven by non-polar interactions. Nonetheless, polar interactions also play important roles in orienting the ligands in the pocket. Sun et al. [8] performed in silico studies on the hemagglutinin protein from the swine-origin influenza virus A, which caused a worldwide pandemic in 2009. Since hemagglutinin is the most important protein for the molecular epidemiology and pathogenesis of the influenza virus, this study should shed new light on the determinants of the virulence and pathogenicity of the virus. $\mathrm{Li}$ and coworkers [9] analyzed the rare codon and mRNA structure of Ustilago maydis CYP51 and performed docking of this protein with the fungicide Tebuconazole. The study may provide the new directions for the development of new antifungal chemicals. Hu et al. [10] studied the interactions between HIV-1 integrase and the inhibitor L708,906 using principal component analysis and dynamical cross-correlation map 
methods. The results of this study indicate that the binding of the inhibitor leads to a decrease of the flexibility of the functional loop regions of the enzyme, and the loss of regular motions and an increase of the correlative motions. He et al. [11] used the FoldX force field to build rapidly models of 420 zinc-finger proteins with DNA-binding affinities.

In the field of methodology studies, Gong et al. [12] reported the development of a holistic molecular docking approach for predicting protein-protein structures and demonstrated, in detail, how to use the programs.

\section{Structural studies of proteins}

Papers in this section range from preliminary crystallographic studies [13] to fully-fledged structural analyses of proteins, to the development of new crystallization techniques.

Bian et al. [14] solved the $1.9 \AA$ resolution crystal structure of the $\mathrm{C}$-terminal part of human galectin- 3 containing a carbohydrate recognition domain in complex with a Thomsen-Friedenreich antigen (TF) derivative. The results of this study showed that contrary to previous observations that the galectin carbohydrate recognition domain only specifically recognizes and binds one carbohydrate ligand, this domain is able to bind two TF antigen molecules. The authors discussed the possible roles of this double binding mode of galectin.

Liu et al. [3] reported the $7.8 \AA$ resolution structure of the hepatitis B core antigen particle truncated at residue 154 by cryo-electron microscopy. Since the C-terminus, which is responsible for the encapsidation of RNA, was removed, the current structure is the empty capsid.

Feng et al. [15] studied the structural basis of thermostability of a histidine-containing phosphocarrier protein (HPr) from Thermoanaerobacter tengcongensis. HPr is a small protein devoid of disulfide bonds. By comparison with homologous proteins from meso- and thermophilic bacteria, the authors proposed that the existence of extra salt bridges may play important roles in the hyperthermophilic properties of HPr.

Wang et al. [16] studied the substrate binding site of group II chaperonin ATcpn $\beta$ from Acidianus tengchongensis based on its crystal structure. This study showed that despite the existence of numerous hydrophilic residues at the binding site, the binding of substrates is still driven by hydrophobic interactions.

Prof. Yin's group [17] at Northwest Polytechnic University has pioneered novel protein crystallization techniques. $\mathrm{He}$ and some of his colleagues reviewed the development of protein screening kits, as well as their experiences in the construction of new kits. This review can serve as a direction for those who plan to design new conditions for their specific proteins.

For techniques in structural studies, Feng et al. [18] re- viewed recent progress in structure determination where NMR spectroscopy and X-ray crystallography were used in combination to solve protein structures. This powerful combined technique approach may prove valuable for solving the structures of complicated protein assemblies.

\section{Functional studies of proteins}

Prof. He's lab at the Institute of Biophysics, Chinese Academy of Sciences, has been engaged in the structural and functional assays of Tau protein, a protein whose hyperphosphorylation is a key marker of Alzheimer's disease. In two papers, he and his colleagues reported the formaldehyde-induced changes of Tau protein, such as the formation of pore-like aggregates [19] and hyperphosphorylation that disrupts its binding to DNA [20]. These behaviors may be pertinent to the roles played by the Tau proteins in the pathology of the Alzheimer's disease. Wu et al. [21] from Fudan University demonstrated that the phosphorylation of the Tau protein when mice were treated with cold water stress was mediated by an excitatory neurotransmission system through ionic excitatory amino acid receptors.

Fan et al. [22] reported the preparation and characterization of a $\mathrm{T} 7$ endonuclease I with a single active domain, which can act as a resolvase to bind and resolve Holliday Junctions. Peng et al. [23] characterized the gene product of human RNF122, which was predicted to contain a RING-H2 domain, and found that the RING domain is required for the apoptosis function of RNF122. This observation may point to the physiological roles of this protein.

The S100 EF-hand $\mathrm{Ca}^{2+}$-binding motif-containing protein Cornulin is a member of a newly identified stress protein family in mammals that defend against environmental stresses and maintain tissue integrity. Li et al. [24] cloned, expressed, purified and assayed this protein, and demonstrated that it undergoes $\mathrm{Ca}^{2+}$-dependent multimerization and the multimerization is required for its full function. This work revealed the importance of the $\mathrm{S} 100$ domain in the functioning of Cornulin.

Zhang et al. [25] assayed the effects of MG132, an inhibitor of the proteasome, and reported that it inhibits Bel-7404 cells growth significantly via both the apoptosis pathway and the related autophagy pathway. Li et al. [26] characterized the protein CT440 in Chlamydia trachomatis-infected cells and found CT440 is an inclusion membrane protein that may play important roles in the pathogenesis of C. trachomatis. Zhao et al. [27] identified a series of immunoreactive proteins of Brucella melitensis that may be used as candidates for vaccine development. Wu et al. [28] characterized the HSV-1 stimulation-related gene 1 (HSRG1) protein and proposed that it probably inhibits viral gene transcriptional elongation by interacting with Cyclin T2. Fang et al. [29] engineered and a fusion protein consisting of an anti-CD20 scFv fragment and lidamycin and characterized 
its antitumor activities. The results of this study indicated that this fusion protein is a potential candidate for tumor-targeted therapy.

For enzymology studies, Zhang et al. [30] revisited the "induced fit" and "lock and key" models that describe enzyme-substrate recognition and binding. Based on their previous observations that the protease I from Eisenia fetida (EfP-I) followed an "induced fit" followed by "lock and key" model, they demonstrated that this "induced fit-lock and key" model could be applied to other enzymes. Xu et al. [31] presented the rigorous derivation of generic rate equations of enzyme-catalyzed reactions that can be used in the kinetic modeling of large-scale metabolic networks.

\section{Reviews}

A series of excellent reviews were published in this period [17,32-51]. Xie et al. [52] reviewed the protective effects of ubiquitin C-terminal hydrolase L1 on neurons. UCH-L1 is critical for the normal morphology and function of the synapses and a mutant form is associated with familial Parkinson's disease. $\mathrm{Li}$ et al. [53] reviewed the effects of SUMOylation on the formation and degradation of promyelocytic leukaemia nuclear bodies (PML NBs). PML NBs are involved in many biological events such as transcriptional regulation, genome stability, response to viral infection, apoptosis and tumor suppression. Guo [54] reviewed the function of a histone trimethylase, PRDM9, in mammalian recombination hotspots. PRDM9 catalyzes H3K4 trimethylation and has transcription factor activity. PRDM9 is only expressed in germ cells entering meiotic prophase in female fetal gonads and in postnatal testis, and its deficiencies results in sterility. Han et al. [55] reviewed a receptor-like kinase subfamily CrRLK1-L, a newly defined family of plant-specific RLK proteins. Members of this family are localized to plasma membranes and their kinase activities are essential to their function as cell surface sensors. Lan et al. [56] reviewed the expression of proteins involved in iron metabolism in kidneys, such as transferring receptor-1, divalent metal transporter-1, ferroportin-1, iron regulatory protein and hepcidin. Kimlicka and van Petegem [57] reviewed recent studies on the structural biology of ryanodine receptors. Ryanodien receptors are large ion channels that facilitate the release of calcium cations from the endoplasmic or sarcoplasmic reticulum. Santella and Chun [58] reviewed the roles played by actin and its interactions with $\mathrm{Ca}^{2+}$ signaling in fertilization. Maki et al. [59] reviewed structural and functional studies on ALG-2, a penta-EF-hand calcium-dependent adaptor protein. A review by Zhang et al. [60] provides readers with an excellent aggregation of human protein databases related to functional proteomics studies.

As part of the celebrations of the 60th Anniversary of the publication of Science China, Science China Life Sciences published a review by Prof. Zhang from the Institute of Biochemistry and Cell Biology, Chinese Academy of Sciences, on the total synthesis of crystalline insulin [61]. This was one of the most important scientific achievements in China, as this achievement represented the first protein to be synthesized in vitro.

1 Yan L C, Su J G, Chen W Z, et al. Study on the characters of different types of amino-acid networks and their relations with protein folding. Prog Biochem Biophys, 2010, 37: 762-768

2 Guo H X, Rao N N, Liu G X, et al. Predicting protein folding rate from amino acid sequence. Prog Biochem Biophys, 2010, 37: 1331-1338

3 Zhang Y, Luo L F. The dynamical contact order: Protein folding rate parameters based on quantum conformational transitions. Sci China Life Sci, 2011, 54: 386-392

4 Chen L N, Wang Q, Shang Y K, et al. Human protein structural interaction network: domain effects on network topology and protein function. Prog Biochem Biophys, 2010, 37: 517-526

5 Wei X L. Construction and analysis for molecular interaction network of cell adhesion. Prog Biochem Biophys, 2011, 38: 347-352

6 Yan J L, Chen Z W, Xu H S, et al. Protein fold recognition by functional domain composition. Prog Biochem Biophys, 2011, 38: $166-172$

7 Cong X J, Tan J J, Liu M, et al. Computational study of binding mode for N-substituted pyrrole derivatives to HIV-1 gp41. Prog Biochem Biophys, 2010, 37: 904-915

8 Sun Y P, Shi Y, Zhang W, et al. In silico characterization of the functional and structural modules of the hemagglutinin protein from the swine-origin influenza virus A (H1N1)-2009. Sci China Life Sci, 2010, 53: 633-642

9 Li S X, Han R, Yuan L L, et al. Analysis of rare codon and mRNA structure about Ustilago maydis CYP51 and molecular docking with fungicide tebuconazole. Prog Biochem Biophys, 2011, 38: 751-758

10 Hu J P, Liu W, Tang D Y, et al. Study on the binding mode and mobility of HIV-1 integrase with L708, 906 inhibitor. Prog Biochem Biophys, 2011, 38: 338-346

11 He Z Y, Mei G, Zhao C P, et al. Potential application of FoldX force field based protein modeling in zinc finger nucleases design. Sci China Life Sci, 2011, 54: 442-449

12 Gong X Q, Liu B, Chang S, et al. A holistic molecular docking approach for predicting protein-protein complex structure. Sci China Life Sci, 2010, 53: 1152-1161

13 Zhang H Y, Liu X Q, Sun Y N, et al. Expression, purification and crystallization of heat shock factor binding protein 1. Prog Biochem Biophys, 2010, 37: 441-444

14 Bian C F, Zhang Y, Li D F, et al. A novel binding pattern unique in two ligands for one carbohydrate recognition domain in galectins. Prog Biochem Biophys, 2011, 38: 810-815

15 Feng C Y, Gao F, Liu Y W, et al. Crystal structure of histidine-containing phosphocarrier protein from Thermoanaerobacter tengcongensis MB4 and the implications for thermostability. Sci China Life Sci, 2011, 54: 513-519

16 Wang L, Zhang K, Fan Z, et al. Substrate binding properties of thermosome ATcpn $\beta$ from Acidianus tengchongensis. Prog Biochem Biophys, 2011, 38: 151-158

17 Zhang X F, Liu J, Guo Y Z, et al. Progresses on developing screening kits for protein crystallization. Prog Biochem Biophys, 2011, 38: 280-287

18 Feng W, Pan L F, Zhang M J. Combination of NMR spectroscopy and X-ray crystallography offers unique advantages for elucidation of the structural basis of protein complex assembly. Sci China Life Sci, 2011, 54: 101-111

19 Naqvi S H, Wang W S, Miao J Y, et al. Pore-like aggregates of Tau protein induced by formaldehyde. Prog Biochem Biophys, 2010, 37 : 1195-1203 
20 Lu J, Miao J Y, Pan R, et al. Formaldehyde-mediated hyperphosphorylation disturbs the interaction between Tau protein and DNA. Prog Biochem Biophys, 2011, 38: 1113-1120

21 Wu F Y, Feng Q, Cheng M, et al. The activation of excitatory amino acid receptors is involved in tau phosphorylation induced by cold water stress. Prog Biochem Biophys, 2010, 37: 510-516

22 Fan S H, Shan L W, Wang B L, et al. Preparation and characterization of T7 endonuclease I with single active domain. Prog Biochem Biophys, 2010, 37: 426-432

23 Peng Z, Ji Z N, Shi T P, et al. RNF122 induces cell apoptosis dependent on its RING domain. Prog Biochem Biophys, 2011, 38 : $816-820$

24 Li G M, Chen Q A, Dai K S, et al. The $\mathrm{Ca}^{2+}$-dependent multimerization S100 domain in Homo sapiens cornulin protects cells from injury. Prog Biochem Biophys, 2011, 38: 239-247

25 Zhang J, Li W, Zhang K J, et al. A study on the mechanism of the inhibition of Bel-7404 hepatocarcinoma cell growth by MG132. Prog Biochem Biophys, 2010, 37: 627-634

26 Li Z Y, Huang Q L, Su S M, et al. Localization and characterization of the hypothetical protein CT440 in Chlamydia trachomatis-infected cells. Sci China Life Sci, 2011, 54: 1048-1054

27 Zhao Z P, Yan F, Ji W H, et al. Identification of immunoreactive proteins of Brucella melitensis by immunoproteomics. Sci China Life Sci, 2011, 54: 880-887

28 Wu W J, Yu X, Li W Z, et al. HSV-1 stimulation-related protein HSRG1 inhibits viral gene transcriptional elongation by interacting with Cyclin T2. Sci China Life Sci, 2011, 54: 359-365

29 Fang H, Miao Q F, Zhang S H, et al. Antitumor effects of an engineered and energized fusion protein consisting of an anti-CD20 scFv fragment and lidamycin. Sci China Life Sci, 2011, 54: 255-262

30 Zhang Z J, Pan R, Zhou Y, et al. "Induced Fit-Lock and Key" model in enzymic reactions. Prog Biochem Biophys, 2011, 38: 418-426

$31 \mathrm{Xu} \mathrm{M} \mathrm{J,} \mathrm{Zhu} \mathrm{X} \mathrm{M,} \mathrm{Lin} \mathrm{P} \mathrm{H,} \mathrm{et} \mathrm{al.} \mathrm{Generic} \mathrm{enzymatic} \mathrm{rate} \mathrm{equation.}$ Prog Biochem Biophys, 2011, 38: 759-767

32 Chen Y F, Li T, An H L, et al. TMEM16A: advances in calcium-activated chloride channel. Prog Biochem Biophys, 2010, 37: 1175-1181

33 Xiao F, Song H T, Wei Q. Structure and function of Kelch proteins in mammals. Prog Biochem Biophys, 2011, 38: 210-217

34 Xiong Y, Gong X W, Jiang Y. Progresses in the studies on kindlin family members. Prog Biochem Biophys, 2010, 37: 1265-1270

35 Wang J L, Wang L S, Liu W F, et al. Research advances on the assembly mode of cellulosomal macromolecular complexes. Prog Biochem Biophys, 2011, 38: 28-35

36 Lu L N, Tang T S, Guo C X. Advances of study on translesion DNA synthesis polymerase kappa in mammalian cells. Prog Biochem Biophys, 2011, 38: 204-209

37 Zhao G J, Lu Z Q, Yao Y M. Research progress of mitofusin-2. Prog Biochem Biophys, 2010, 37: 252-260

38 Tan Y C, Wang Q J, Zhao G P, et al. Protein post-translational modification in prokaryotes. Prog Biochem Biophys, 2011, 38: 197-203

39 Tu L H, Liu H P, Luo J. A new family of regulators of calcineurin (RCANs). Prog Biochem Biophys, 2010, 37: 22-28

$40 \mathrm{Li} \mathrm{Y} \mathrm{M,} \mathrm{Ji} \mathrm{G} \mathrm{J.} \mathrm{Evolution} \mathrm{in} \mathrm{research} \mathrm{of} \mathrm{ryanodine} \mathrm{receptors} \mathrm{and} \mathrm{its}$ subtype 2 regulators. Prog Biochem Biophys, 2011, 38: 408-417
41 Peng Q, Chen W C, Liu X G. Advances in relationship between deacetylase (Sirtuin) and aging. Prog Biochem Biophys, 2010, 37 : 1271-1277

42 Zhang Y J, Jiang J H, Xie J, et al. Lysyl oxidases related to human diseases. Prog Biochem Biophys, 2011, 38: 389-399

43 Zhou X J, Hu Y, Liu P. Advances in relationship between brain-derived neurotrophic factor and depressive disorder. Prog Biochem Biophys, 2011, 38: 1085-1090

44 Xu S J, Liu G L. Recent progress of mitochondrial dysfunction induced by $\beta$-amyloid protein. Prog Biochem Biophys, 2010, 37: 589-593

45 Chen J W, Zuo Q H, Ji L, et al. The research advances in ubiquitin-independent degradation of proteins, Prog Biochem Biophys, 2011, 38: 593-603

46 Wang C J, Lin J, Zhang J J. Progress in the study of prokaryotic ubiquitin-like protein (Pup)-proteasome system. Prog Biochem Biophys, 2011, 38: 1091-1098

47 Shao C, Wang Y, Gao Y H. Applications of urinary proteomics in biomarker discovery. Sci China Life Sci, 2011, 54: 409-417

48 Li H M, Wan X R, He S G. Advances in plant aquaporins. Prog Biochem Biophys, 2010, 37: 29-35

49 Zhang Y X, Liu J G, Chai T Y, et al. Advances in the uptake and translocation of silicon in plants. Prog Biochem Biophys, 2011, 38: 400-407

50 Wang Y F, Yu W C, Zhou F J, et al. Applications of terahertz(THz) spectroscopy in biological macromolecules. Prog Biochem Biophys, 2010, 37: 484-489

51 Sun Y P, Gao G F. Structural and functional studies of the innate immune effector Mx proteins: a review. Prog Biochem Biophys, 2010, 37: 699-706

52 Xie M, Chen Q C, Liao X M. The protective effects of ubiquitin C-terminal hydrolase L1 on neurons. Prog Biochem Biophys, 2010, 37: 1054-1058

53 Li Q, Pan C, Lei M. SUMOylation and PML NBs formation. Prog Biochem Biophys, 2010, 37: 707-712

54 Guo X Q. The function of PRDM9 on mammalian recombination hotspots. Prog Biochem Biophys, 2010, 37: 929-931

55 Han Y F, Yang Q, Zhang S W, et al. Receptor-like kinase CrRLK1-L subfamily: novel motifs in extracellular domain and biological functions in plants. Prog Biochem Biophys, 2011, 38: 891-899

56 Lan Y, Qian Z M, Ke Y. Expression and function of iron metabolism proteins in the kidney. Prog Biochem Biophys, 2011, 38: 113-118

57 Kimlicka L, van Petegem F. The structural biology of ryanodine receptors. Sci China Life Sci, 2011, 54: 712-724

58 Santella L, Chun J T. Actin, more than just a housekeeping protein at the scene of fertilization. Sci China Life Sci, 2011, 54: 733-743

59 Maki M, Suzuki H, Shibata H. Structure and function of ALG-2, a penta-EF-hand calcium-dependent adaptor protein. Sci China Life Sci, 2011, 54: 770-779

60 Zhang Y Q, Zhu Y P, He F C. An overview of human protein databases and their application to functional proteomics in health and disease. Sci China Life Sci, 2011, 54: 988-998

61 Zhang Y S. The first protein ever synthesized in vitro-A personal reminiscence of the total synthesis of crystalline insulin. Sci China Life Sci, 2010, 53: 16-18

Open Access This article is distributed under the terms of the Creative Commons Attribution License which permits any use, distribution, and reproduction in any medium, provided the original author(s) and source are credited. 